\title{
A New Multilevel Thresholding Method Using Swarm Intelligence Algorithm for Image Segmentation
}

\author{
Sathya P. Duraisamy, Ramanujam Kayalvizhi \\ ${ }^{1}$ The Department of Electrical Engineering, Faculty of Engineering and Technology, Annamalai University, Chidambaram, India; \\ ${ }^{2}$ Department of Instrumentation Engineering, Faculty of Engineering and Technology, Annamalai University, Chidambaram, India. \\ Email: pd.sathya@yahoo.in, mithuvig.knr@gmail.com
}

Received June $11^{\text {th }}, 2010$; revised June $29^{\text {th }}, 2010$; accepted July $20^{\text {th }}, 2010$.

\begin{abstract}
Thresholding is a popular image segmentation method that converts gray-level image into binary image. The selection of optimum thresholds has remained a challenge over decades. In order to determine thresholds, most methods analyze the histogram of the image. The optimal thresholds are often found by either minimizing or maximizing an objective function with respect to the values of the thresholds. In this paper, a new intelligence algorithm, particle swarm optimization (PSO), is presented for multilevel thresholding in image segmentation. This algorithm is used to maximize the Kapur's and Otsu's objective functions. The performance of the PSO has been tested on ten sample images and it is found to be superior as compared with genetic algorithm (GA).
\end{abstract}

Keywords: Image Segmentation, Multilevel Thresholding, Particle Swarm Optimization

\section{Introduction}

In many image processing applications, the gray levels of pixels belonging to an object are substantially different from those belonging to the background. As such, thresholding techniques can be used to extract the objects from their background. Indeed, thresholding is a major operation in many image processing applications such as document processing, image compression, particle counting, cell motion estimation and object recognition. The effect of many image processing applications strongly depends on the effect of image thresholding.

Thresholding techniques provide an efficient way, in terms of both the implementation simplicity and the processing time to perform image segmentation. However, the automatic selection of a robust optimum threshold has remained a challenge in image segmentation. Besides being segmentation on its own, thresholding is frequently used as one of the steps in many advanced segmentation methods. In these applications, thresholding is not applied on the original images, but applied in a space generated by the segmentation method. For example, in fuzzy connectedness segmentation [1], a threshold is applied on the strength of connectedness among image elements to produce a final segmentation. Thus, the methods to de- termine effective thresholds have wide-spread applications. However, automatic determination of the optimum threshold value is often a difficult task. While a number of approaches for automatic threshold determination have been proposed over the past several decades, applying new ideas and concepts to image thresholding remains an interesting and challenging research area.

Excellent reviews on early thresholding methods can be found in $[2,3]$, whereas the latest development in this topic was summarized in [4]. Comparative performance studies of global thresholding techniques were presented by Lee et al. [5]. Otsu [6] proposed a method that maximizes between-class variance. Tao et al. [7] proposed a thresholding method for object segmentation based on fuzzy entropy theory and ant colony optimization algorithm. An image histogram thresholding approaches using fuzzy sets was proposed by Tobias and Seara [8].

Methods based on optimizing an objective function include maximization of posterior entropy to measure homogeneity of segmented Classes [9-11], maximization of the measure of seperability on the basis of betweenclass variance [6], thresholding based on index of fuzziness and fuzzy similarity measure $[12,13]$, minimization of Bayesian error [14,15], etc. several such methods have originally been developed for bi-level thresholding and 
later extended to multilevel thresholding.

Bi-level thresholding divides the pixel into two groups, one including those pixels with gray levels above a certain threshold, the other including the rest. Multilevel thresholding divides the pixels into several groups; the pixels of the same group have gray levels within a specified range. However the problem gets more complex when the segmentation is achieved with greater details by employing multilevel thresholding. Then the image segmentation problem becomes a multiclass classification problem where pixels having gray levels within a specified range are grouped into one class. Usually it is not simple to determine exact locations of distinct valleys in a multimodal histogram of an image, that can segment the image efficiently and hence the problem of multilevel thresholding is regarded as an important area of research interest among the research communities worldwide.

A great number of thresholding methods of parametric or non-parametric type have been proposed in order to perform bi-level thresholding [16] and later extended to multilevel thresholding [17]. In [18], the Otsu's function is modified by a fast recursive algorithm along with a look-up-table for multilevel thresholding. In [19], Lin has proposed a fast thresholding computation using Otsu's function. Another fast multilevel thresholding technique has been proposed by Yin [20].

In recent years, several heuristic optimization techniques such as differential evolution (DE), Ant Colony Optimization (ACO) and Genetic Algorithms (GA) were introduced into the field of image segmentation because of their fast computing ability. Erik Cuevas et al. [21] applied the differential evolution (DE) algorithm to solve the multilevel thressholding problem. The algorithm fills the 1-D histogram of the image using a mix of Gaussian functions whose parameters are calculated using the differential evolution method. Each Gaussian function approximating the histogram represents a pixel class and therefore a threshold point. Tao et al. [22] proposed the Ant Colony Optimization (ACO) algorithm to obtain the optimal parameters of the entropy-based object segmentation approach.

Several techniques using genetic algorithms (GAs) have also been proposed to solve the multilevel thresholding problem [23,24]. Yin [23] introduced a neighborhood searching strategy in to the GA to speed up the multilevel thresholds optimization. Though GA-based approaches perform well for complex optimization problems, recent research has identified certain deficiencies [25], particularly for problems in which variables are highly correlated. In such cases, the GA crossover and mutation operators do not generate individuals with better fitness of offspring as the chromosomes in the population pool have some structure towards the end of the search.
PSO, first introduced by Kennedy and Eberhart [26] is a flexible, robust, population based stochastic search/optimization algorithm with inherent parallelism. This method has gained popularity over its competitors and is increasingly gaining acceptance for solving many image processing problems [27-29]. Compared with other population-based stochastic optimization methods such as DE, ACO and GA, PSO gives superior search performance with faster and more stable convergence rates [26].

This paper presents a new optimal multilevel thresholding algorithm; Particle Swarm Optimization (PSO) for solving the multilevel thresholding problem in image segmentation. The validity of the proposed method is tested on ten sample images and compared with the GA method.

\section{Problem Formulation}

In this paper, two broadly used optimal thresholding methods namely entropy criterion (Kapur's) method and between-class variance (Otsu's) method are used.

Kapur has developed the algorithm for bi-level thresholding and this bi-level thresholding can be described as follows:

Let there be L gray levels in a given image and these gray levels are in a given image and these gray levels are in the range $\{0,1,2, \ldots \ldots \ldots,(L-1)\}$. Then one can define $\mathrm{P}_{i}=h(i) / \mathrm{N},(0 \leq i \leq(L-1))$ where $h(i)$ denotes number of pixels for the corresponding gray-level $\mathrm{L}$ and $\mathrm{N}$ denotes total number of pixels in the image which is equal to $\sum_{i=0}^{L-1} h(i)$.

Then the objective is to maximize the fitness function

$$
f(t)=H_{0}+H_{1}
$$

where $H_{0}=\sum_{i=0}^{t-1} \frac{P_{i}}{w_{0}} \operatorname{In} \frac{P_{i}}{w_{0}}, \quad w_{0}=\sum_{i=0}^{t-1} P_{i}$ and

$$
H_{1}=-\sum_{i=t}^{L-1} \frac{P_{i}}{w_{1}} \operatorname{In} \frac{P_{i}}{w_{1}}, \quad w_{1}=\sum_{i=t}^{L-1} P_{i}
$$

The optimal threshold is the gray level that maximizes Equation (1). This Kapur's entropy criterion method tries to achieve a centralized distribution for each histogram-based segmented region of the image.

This Kapur's entropy criterion method has also been extended to multilevel thresholding and can be described as follows: The optimal multilevel thresholding problem can be configured as a m-dimensional optimization problem, for determination of $\mathrm{m}$ optimal thresholds for a given image $\left[t_{1}, t_{2} \ldots t_{\mathrm{m}}\right]$, where the aim is to maximize the objective function:

$$
f\left(\left[t_{1}, t_{2}, \ldots . . t_{\mathrm{m}}\right]\right)=H_{0}+H_{1}+H_{2}+\ldots .+H_{\mathrm{m}}
$$

where 


$$
\begin{gathered}
H_{0}=\sum_{i=0}^{t_{1}-1} \frac{P_{i}}{w_{0}} \operatorname{In} \frac{P_{i}}{w_{0}}, \quad w_{0}=\sum_{i=0}^{t_{1}-1} P_{i} \\
H_{1}=-\sum_{i=t_{1}}^{t_{2}-1} \frac{P_{i}}{w_{1}} \operatorname{In} \frac{P_{i}}{w_{1}}, \quad w_{1}=\sum_{i=t_{1}}^{t_{2}-1} P_{i} \\
H_{2}=-\sum_{i=t_{2}}^{t_{3}-1} \frac{P_{i}}{w_{2}} \operatorname{In} \frac{P_{i}}{w_{2}}, \quad w_{2}=\sum_{i=t_{2}}^{t_{3}-1} P_{i}, \ldots . . \\
H_{m}=-\sum_{i=t_{m}}^{L-1} \frac{P_{i}}{w_{m}} \operatorname{In} \frac{P_{i}}{w_{m}}, \quad w_{m}=\sum_{i=t_{m}}^{L-1} P_{i} .
\end{gathered}
$$

As Kapur based entropy criterion method, the Otsu based between-class variance method has also been employed in determining whether the optimal thresholding can provide histogram-based image segmentation with satisfactory desired. The Otsu based between-class variance algorithm can be described as follows:

If an image can be divided into two classes, $C_{0}$ and $C_{1}$, by a threshold at a level $\mathrm{t}$, class $C_{0}$ contains the gray levels from 0 to $t-1$ and class $C_{1}$ consists of the other gray levels with $t$ to $L-1$. Then, the gray level probabilities ( $w_{0}$ and $\left.w_{1}\right)$ distributions for the two classes are as follows:

$$
C_{0}: \frac{P_{0}}{w_{0}}, \ldots \ldots \frac{P_{t-1}}{w_{0}} \text { and } C_{1}: \frac{P_{t}}{w_{1}}, \ldots \ldots \frac{P_{L-1}}{w_{1}} .
$$

where, $w_{0}=\sum_{i=0}^{t-1} P_{i}$ and $w_{1}=\sum_{i=t}^{L-1} P_{i}$

Mean levels $\mu_{0}$ and $\mu_{1}$ for classes $C_{0}$ and $C_{1}$ are as follows:

$$
\mu_{0}=\sum_{i=0}^{t-1} \frac{i \times P_{i}}{w_{0}}, \quad \mu_{1}=\sum_{i=t}^{L-1} \frac{i \times P_{i}}{w_{1}} .
$$

Let $\mu_{\mathrm{T}}$ be the mean intensity for the whole image, it is easy to show that

$$
w_{0} \mu_{0}+w_{1} \mu_{1}=\mu_{T} \text { and } w_{0}+w_{1}=1
$$

Using discriminant analysis, Otsu based between-class variance thresholded image can be defined as follows:

$$
f(t)=\sigma_{0}+\sigma_{1}
$$

where $\sigma_{0}=w_{0}\left(\mu_{0}-\mu_{T}\right)^{2}$ and $\sigma_{1}=w_{1}\left(\mu_{1}-\mu_{T}\right)^{2}$

For bi-level thresholding, Otsu selects an optimal threshold $t^{*}$ that maximizes the between-class variance $f(t)$;

that is

$$
t^{*}=\arg \max \{f(t)\} \quad 0 \leq t \leq L-1
$$

The above formula can be easily extended to multilevel thresholding of an image. Assuming that there are $\mathrm{m}$ thresholds, $\left(t_{0}, t_{1}, \ldots, t_{\mathrm{m}}\right)$, which divide the original image into m classes: $C_{0}$ for $\left[0, \ldots, t_{1}-1\right], \mathrm{C}_{1}$ for $\left[t_{1}, \ldots\right.$, $\left.t_{2}-1\right] \ldots .$. and $C_{\mathrm{m}}$ for $\left[t_{\mathrm{m}}, \ldots, L^{-1}\right]$, the optimal thresholds $\left(t_{0}^{*}, t_{1}^{*}, \ldots ., t_{m}^{*}\right)$ are chosen by maximizing $f(t)$ as follows:

$$
\left(t_{0}^{*}, t_{1}^{*}, \ldots, t_{m}^{*}\right)=\arg \max \{f(t)\} \quad 0 \leq t_{1} \leq \ldots . \leq t_{m} \leq L-1
$$

where $f(t)=\sigma_{0}+\sigma_{1}+\sigma_{2} \ldots . .+\sigma_{m}$

with $\sigma_{0}=w_{0}\left(\mu_{0}-\mu_{T}\right)^{2}$,

$$
\begin{aligned}
& \sigma_{1}=w_{1}\left(\mu_{1}-\mu_{T}\right)^{2}, \\
& \sigma_{2}=w_{2}\left(\mu_{2}-\mu_{T}\right)^{2}, \ldots . \\
& \sigma_{m}=w_{m}\left(\mu_{m}-\mu_{T}\right)^{2} .
\end{aligned}
$$

The Kapur and Otsu methods have been proven as an efficient method for bi-level thresholding in image segmentation. However, when these methods are extended to multilevel thresholding, the computation time grows exponentially with the number of thresholds. It would limit the multilevel thresholding applications. To overcome the above problem, this paper proposes the Kapur and Otsu based PSO algorithm for solving multilevel thresholding problem. The aim of this proposed method is to maximize the Kapur's and Otsu's objective function using Equations (2) and (3).

\section{Particle Swarm Optimization (PSO)}

PSO is a simple end efficient population-based optimization method proposed by Kennedy and Eberhart [24]. It is motivated by social behavior of organisms such as fish schooling and bird flocking. In PSO, potential solutions called particles fly around in a multi-dimensional problem space. Population of particles is called swarm. Each particle in a swarm flies in the search space towards the optimum solution based on its own experience, experience of nearby particles, and global best position among particles in the swarm.

\subsection{Advantages of PSO}

1) PSO is easy to implement and only few parameters have to be adjusted.

2) Unlike the GA, PSO has no evolution operators such as crossover and mutation.

3) In GAs, chromosomes share information so that the whole population moves like one group, but in PSO, only global best particle (gbest) gives out information to the others. It is more robust than GAs.

4) PSO can be more efficient than GAs; that is, PSO often finds the solution with fewer objective function evaluations than that required by GAs.

Unlike GAs and other heuristic algorithms, PSO has the 
flexibility to control the balance between global and local exploration of the search space.

\subsection{PSO Algorithm}

Let $X$ and $V$ denote the particle's position and its corresponding velocity in search space respectively. At iteration $\mathrm{K}$, each particle $i$ has its position defined by $X_{\mathrm{i}}^{\mathrm{K}}=$ $\left[X_{i, 1}, X_{i, 2} \ldots X_{i, \mathrm{~N}}\right]$ and a velocity is defined as $V_{i}^{\mathrm{K}}=\left[V_{i, 1}\right.$, $\left.V_{i, 2} \ldots \ldots V_{i, \mathrm{~N}}\right]$ in search space $\mathrm{N}$. Velocity and position of each particle in the next iteration can be calculated as

$$
\begin{gathered}
V_{i, \mathrm{n}}{ }^{\mathrm{k}+1}=W \times V_{i, \mathrm{n}}{ }^{\mathrm{k}}+C_{1} \times \operatorname{rand}_{1} \times\left(\text { pbest }_{i, \mathrm{n}}-X_{i, \mathrm{n}}{ }^{\mathrm{k}}\right)+C_{2} \times \\
\operatorname{rand}_{2} \times\left(\text { gbest }_{\mathrm{n}}-X_{i, \mathrm{n}}{ }^{\mathrm{k}}\right) \\
i=1,2 \ldots \ldots \ldots \mathrm{m} \\
n=1,2 \ldots \ldots \ldots \mathrm{N} \\
X_{i, \mathrm{n}}{ }^{\mathrm{k}+1}=X_{i, \mathrm{n}}{ }^{\mathrm{k}}+V_{i, \mathrm{n}}{ }^{\mathrm{k}+1} \text { if } X_{\min , \mathrm{i}, \mathrm{n}} \leq X_{\mathrm{i}}^{\mathrm{k}+1} \leq X_{\max \text { i,n }} \\
=X_{\min \text { i,n }} \text { if } X_{i, \mathrm{n}}{ }^{\mathrm{k}+1}<X_{\min i, \mathrm{n}} \\
=X_{\max \text { i,n }} \text { if } X_{i, \mathrm{n}}{ }^{\mathrm{k}+1}>X_{\max i, \mathrm{n}}
\end{gathered}
$$

The inertia weight $\mathrm{W}$ is an important factor for the PSO's convergence. It is used to control the impact of previous history of velocities on the current velocity. A large inertia weight factor facilitates global exploration (i.e., searching of new area) while small weight factor facilitates local exploration. Therefore, it is better to choose large weight factor for initial iterations and gradually reduce weight factor in successive iterations. This can be done by using

$$
W=W_{\max }-\left(W_{\max }-W_{\min }\right) \times \text { Iter/Iter }_{\max }
$$

Where $W_{\max }$ and $W_{\min }$ are initial and final weight respectively, Iter is current iteration number and Iter max $_{\text {is }}$ maximum iteration number.

Acceleration constant $C_{1}$ called cognitive parameter pulls each particle towards local best position whereas constant $C_{2}$ called social parameter pulls the particle towards global best position. The particle position is modified by Equation (4). The process is repeated until stopping criterion is reached.

\section{Implementation of PSO for Multilevel Thresholding Problem}

This paper presents a quick solution to the multilevel image thresholding problems using the PSO algorithm. The number of threshold levels is the dimension of the problem. For example, if there are ' $\mathrm{m}$ ' threshold levels, the ith particle is represented as follows:

$$
X_{\mathrm{i}}=\left(X_{\mathrm{i} 1}, X_{\mathrm{i} 2}, \ldots \ldots \ldots, X_{\mathrm{im}}\right)
$$

Its implementation consists of the following steps.

Step 1. Initialization of the swarm: For a population size $\mathrm{p}$, the particles are randomly generated between the minimum and the maximum limits of the threshold values.

Step 2. Evaluation of the objective function: The ob- jective function values of the particles are evaluated using the objective functions given by Equation (2) or (3).

Step 3. Initialization of pbest and gbest: The objective values obtained above for the initial particles of the swarm are set as the initial pbest values of the particles. The best value among all the pbest values is identified as gbest.

Step 4. Evaluation of velocity: The new velocity for each particle is computed using Equation (4).

Step 5. Update the swarm: The particle position is updated using Equation (5). The values of the objective function are calculated for the updated positions of the particles. If the new value is better than the previous pbest, the new value is set to pbest. Similarly, gbest value is also updated as the best pbest.

Step 6. Stopping criteria: If the stopping criteria are met, the positions of particles represented by gbest are the optimal threshold values. Otherwise, the procedure is repeated from step 4 .

\section{Experimental Results and Discussions}

In this section, the effectiveness and feasibility of the proposed PSO method for multilevel thresholding is demonstrated. Comparisons are performed with the results provided by GA based multilevel thresholding method. Tables 1 and 2 represent the various parameters chosen for the implementation of GA and PSO algorithms respectively. Ten well-known images namely lena, pepper, baboon, hunter, map, cameraman, living room, house, airplane and butterfly are taken as the test images, and are gathered with their histograms in Figure 1.

The quality of the thresholded images for Kapur based

Table 1. Parameters chosen for GA implementation

\begin{tabular}{cc}
\hline Parameter & Value \\
\hline Population size & 20 \\
No. of Iterations & 100 \\
Crossover probability & 0.9 \\
Mutation probability & 0.1 \\
Selection operator & Roulette Wheel Selection \\
\hline
\end{tabular}

Table 2. Parameters chosen for PSO implementation

\begin{tabular}{cc}
\hline Parameter & Value \\
\hline Swam Size & 20 \\
No. of Iterations & 100 \\
$\mathrm{~W}_{\max }, \mathrm{W}_{\min }$ & $0.4,0.1$ \\
$\mathrm{C}_{1}, \mathrm{C}_{2}$ & 2 \\
\hline
\end{tabular}




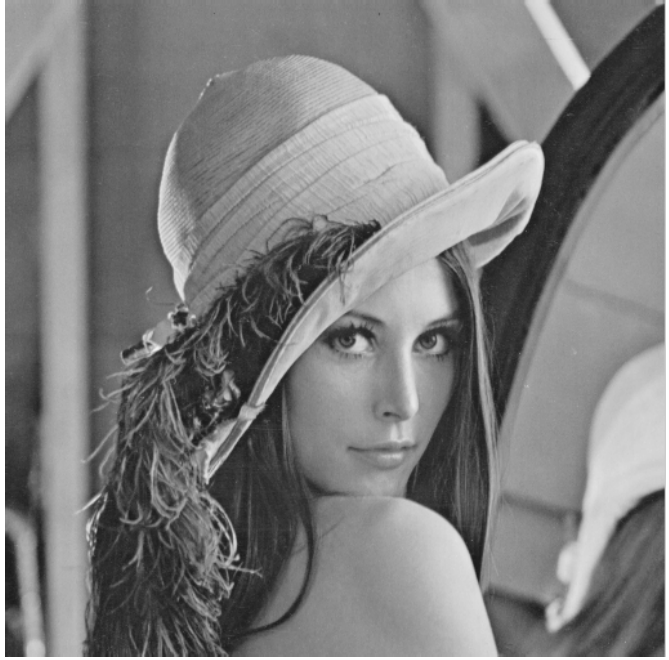

(a)

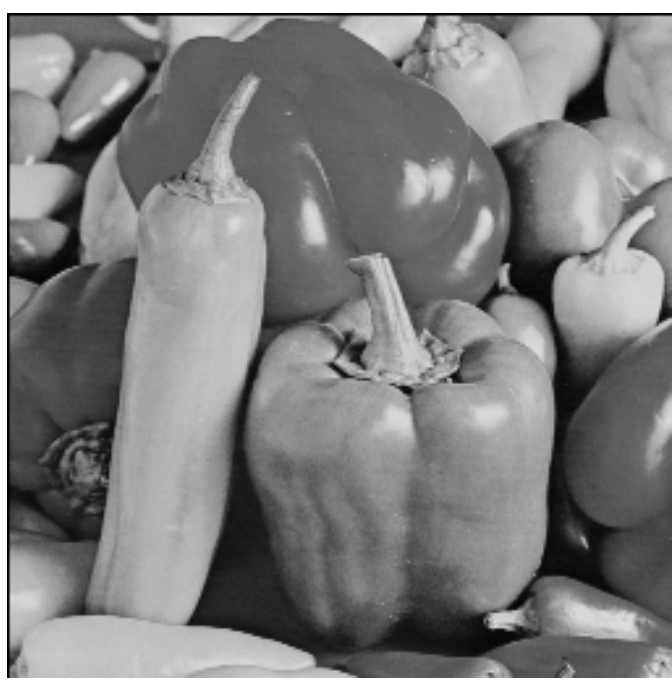

(b)

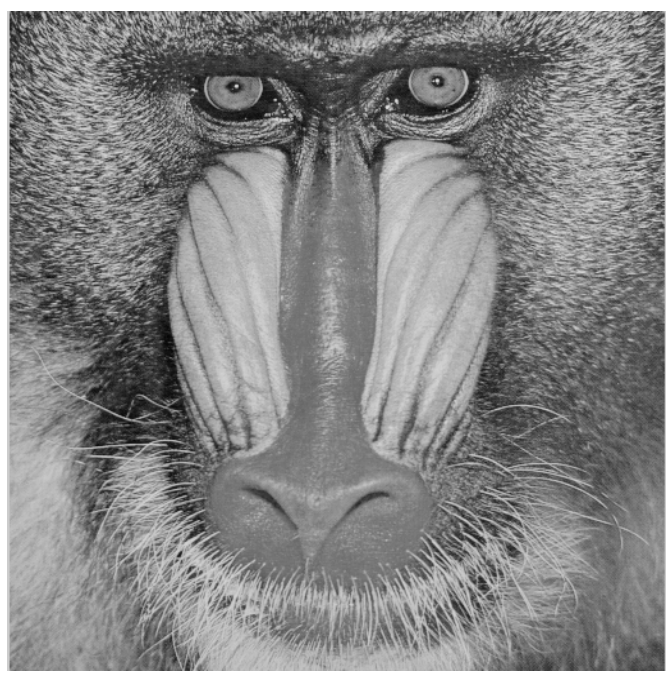

(c)

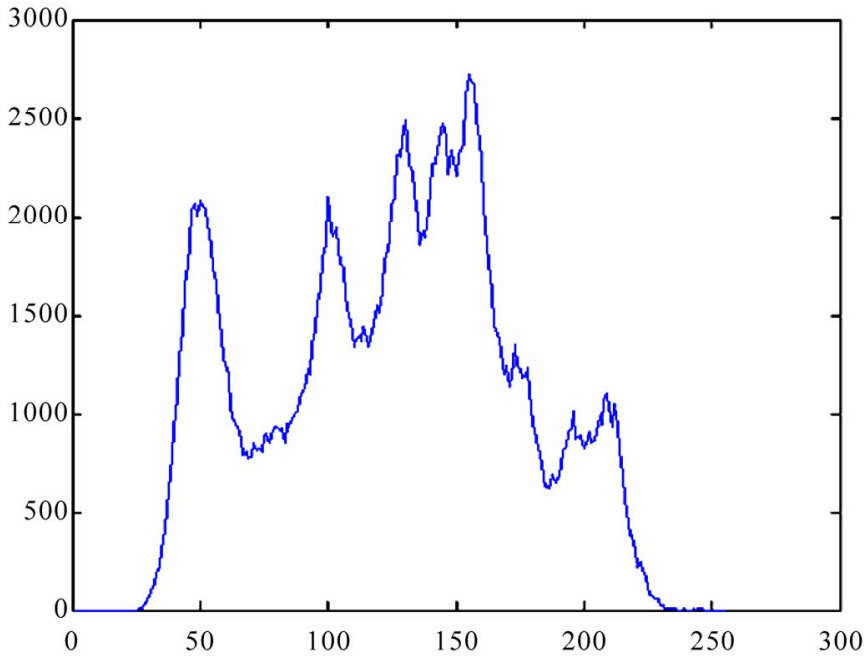

(a')

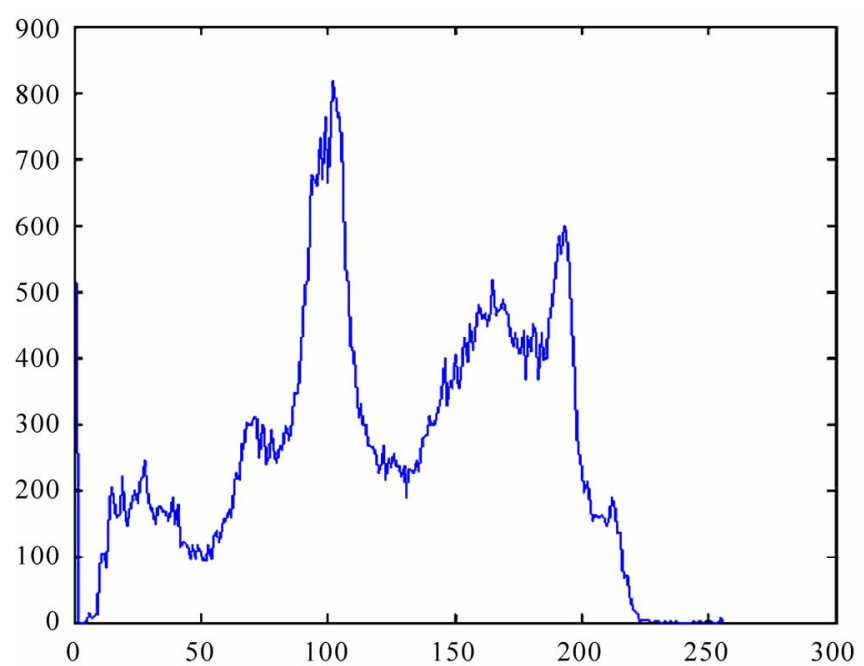

(b')

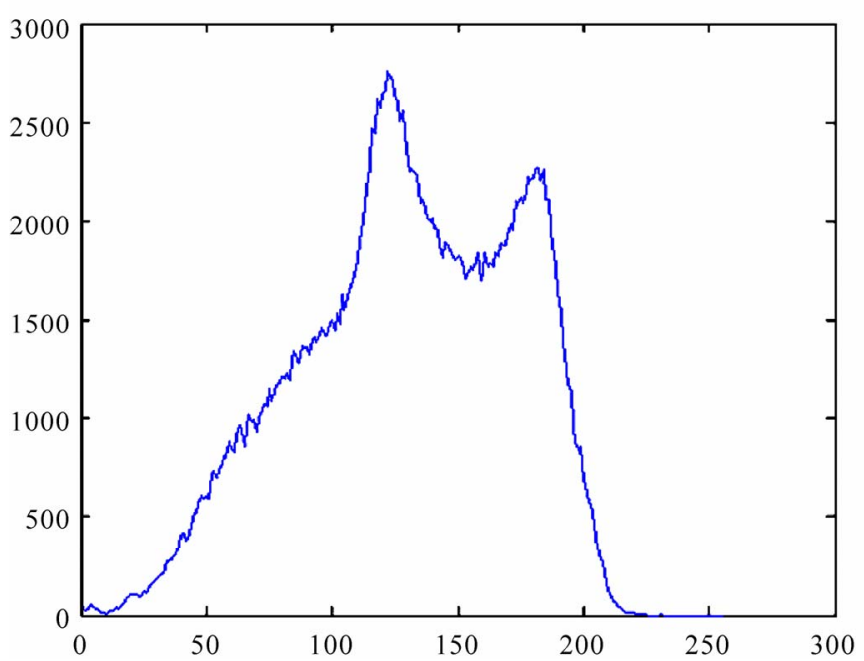

(c') 


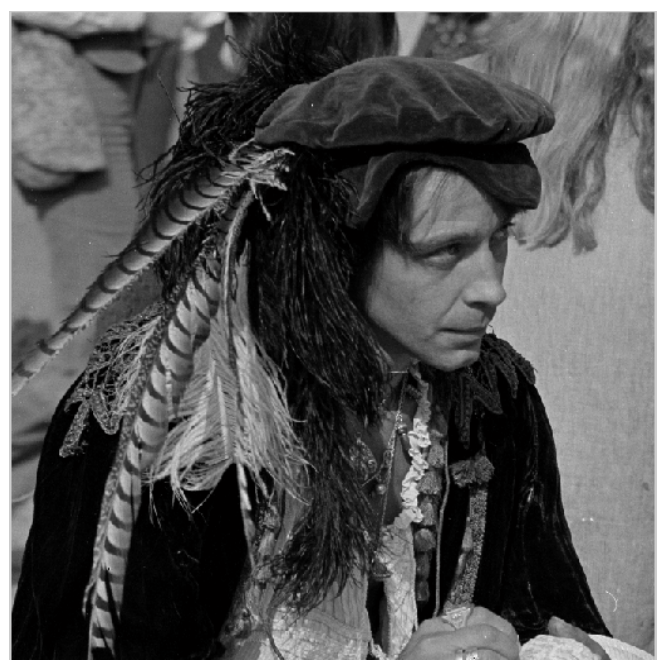

(d)

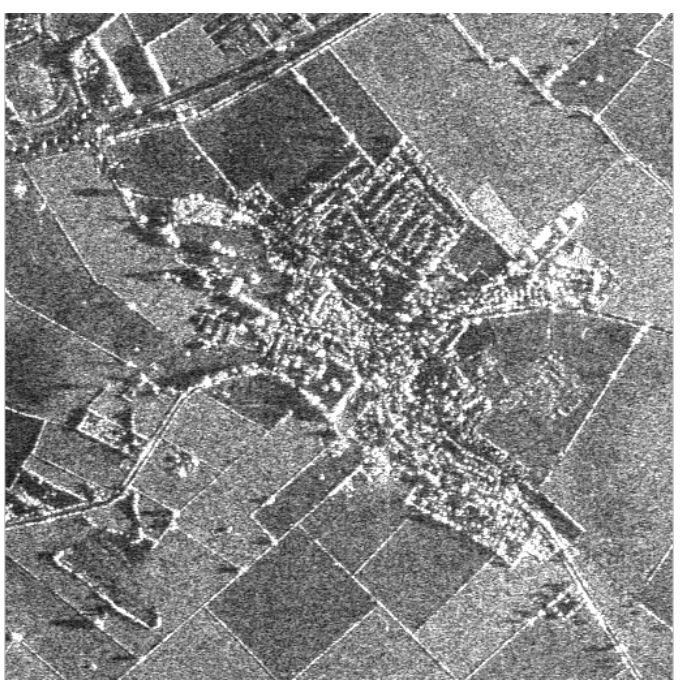

(e)

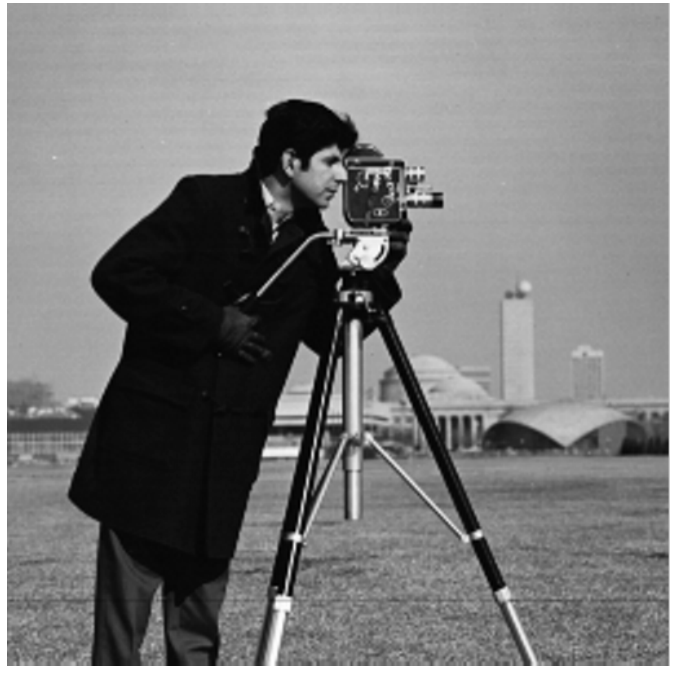

(f)

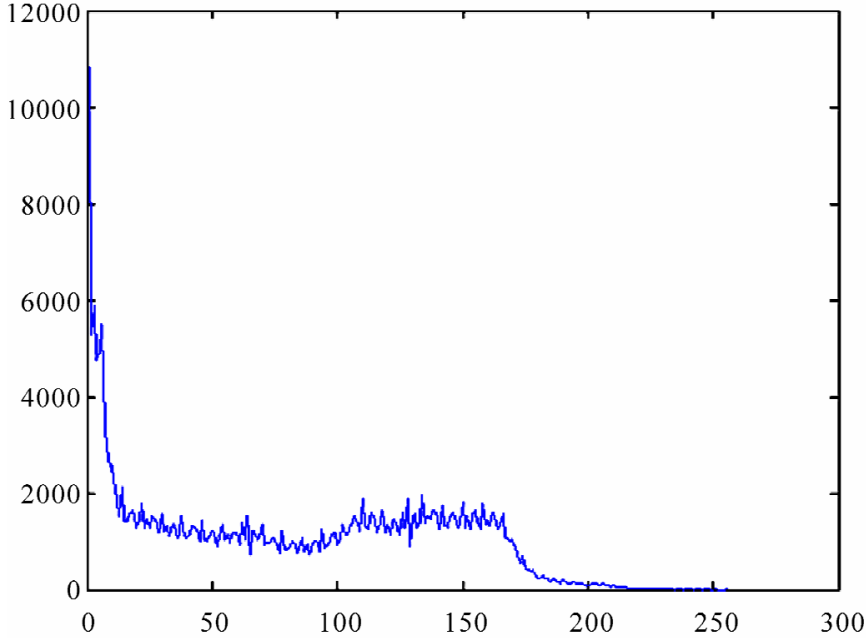

(d')

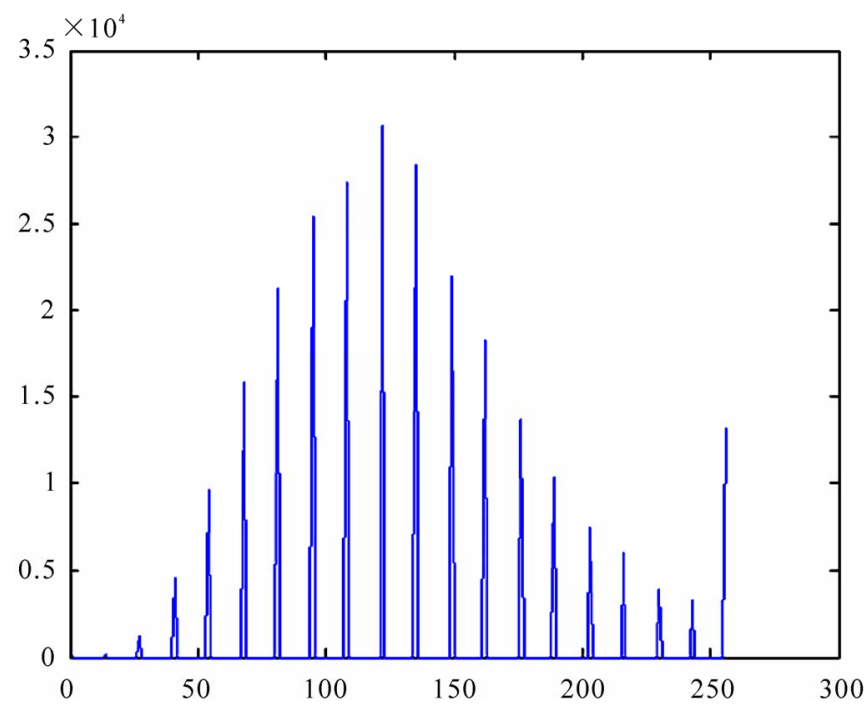

(e')

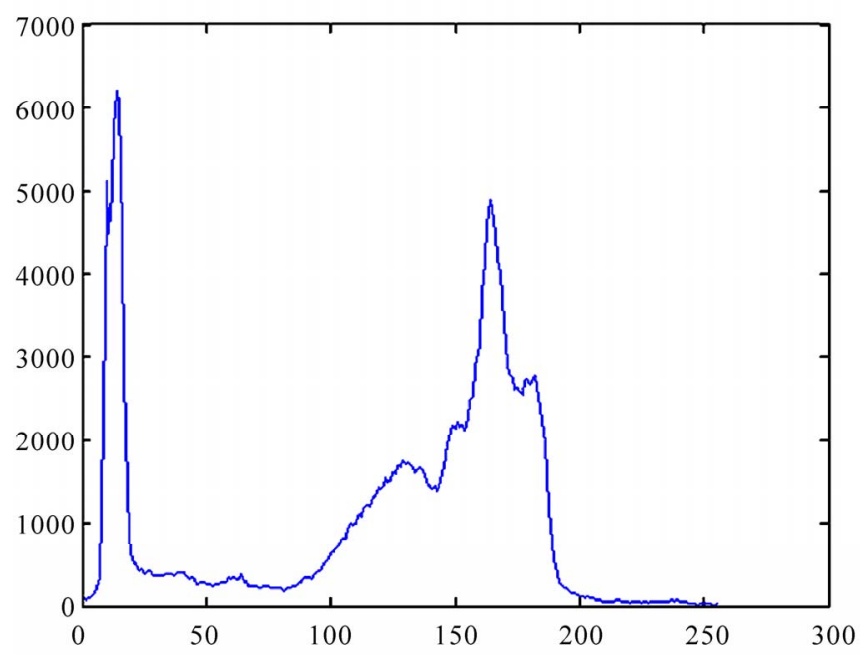

(f') 


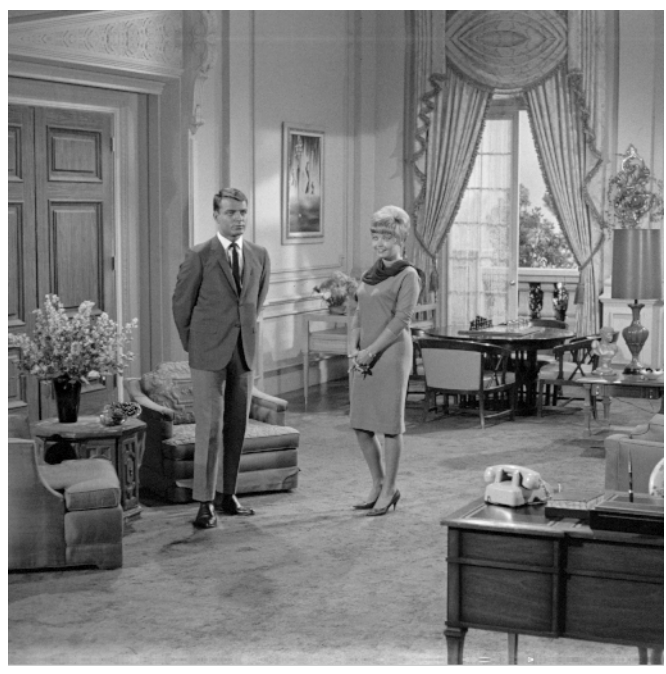

(g)

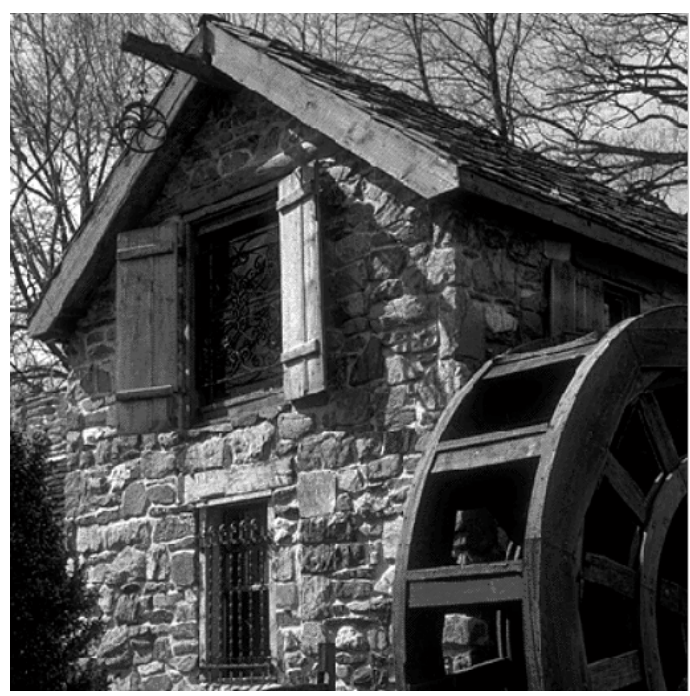

(h)

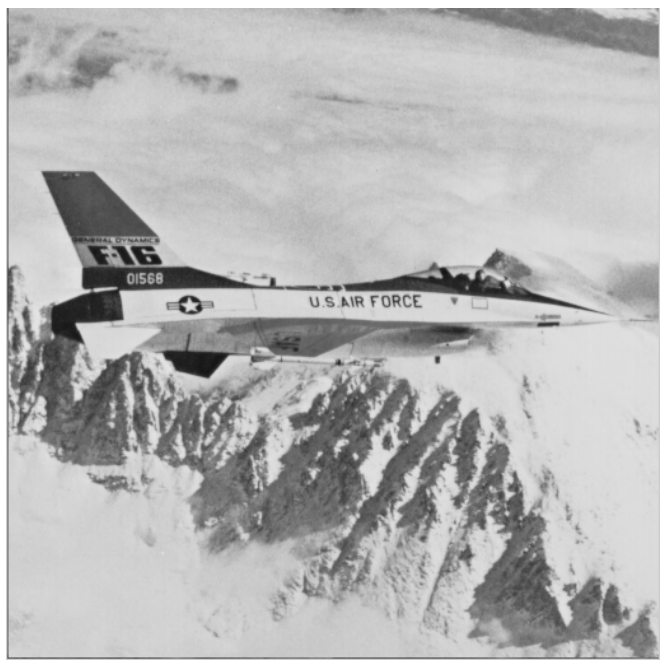

(i)

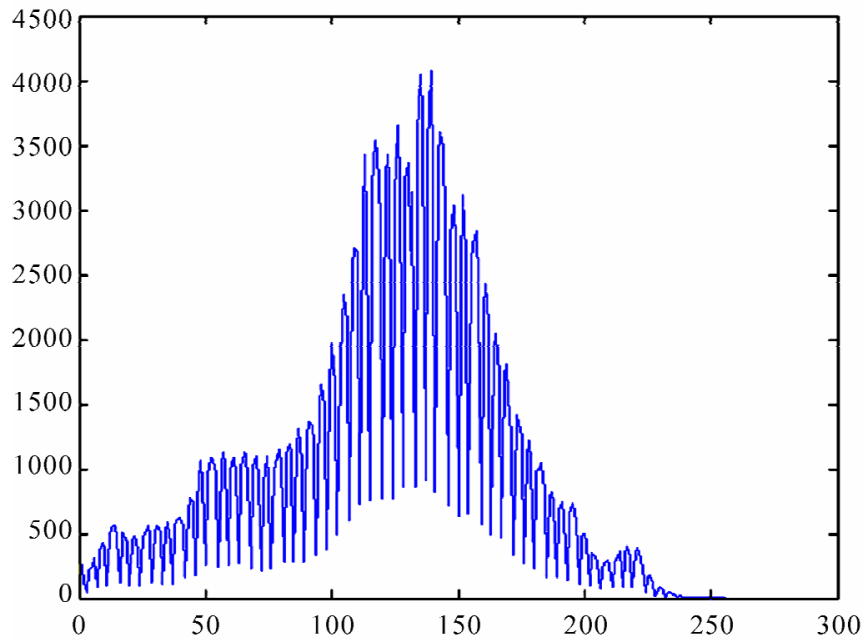

(g')

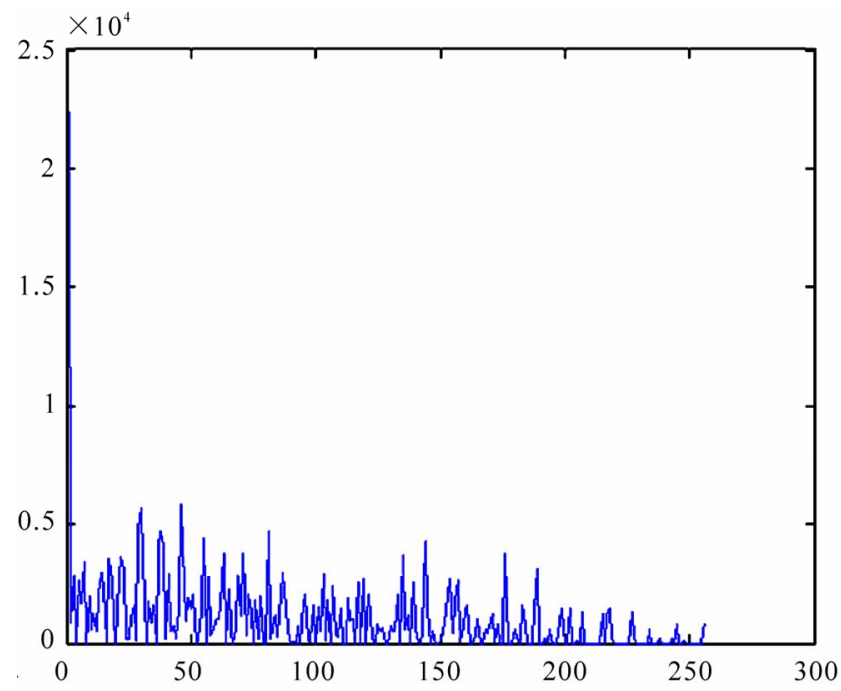

(h')

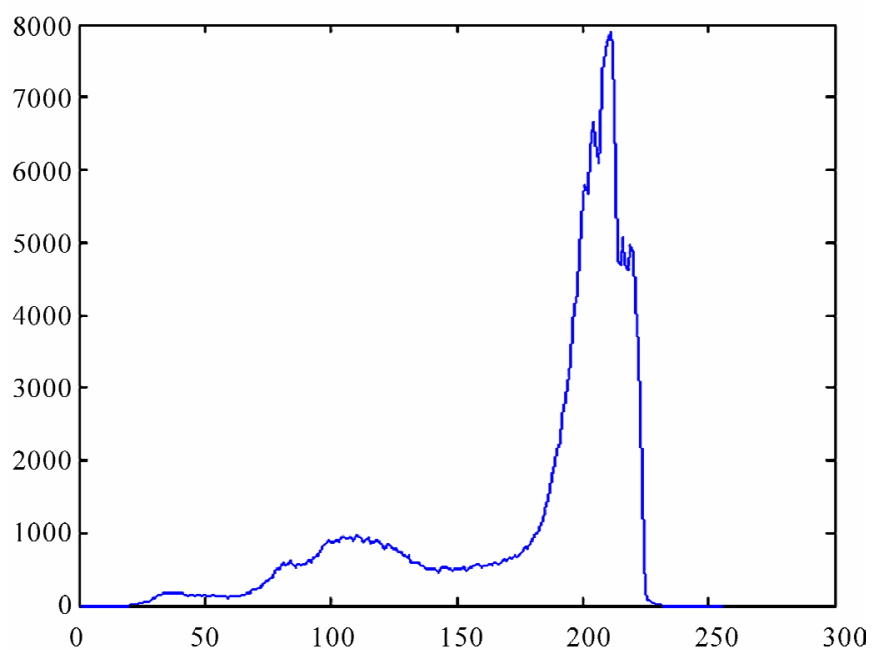

(i') 


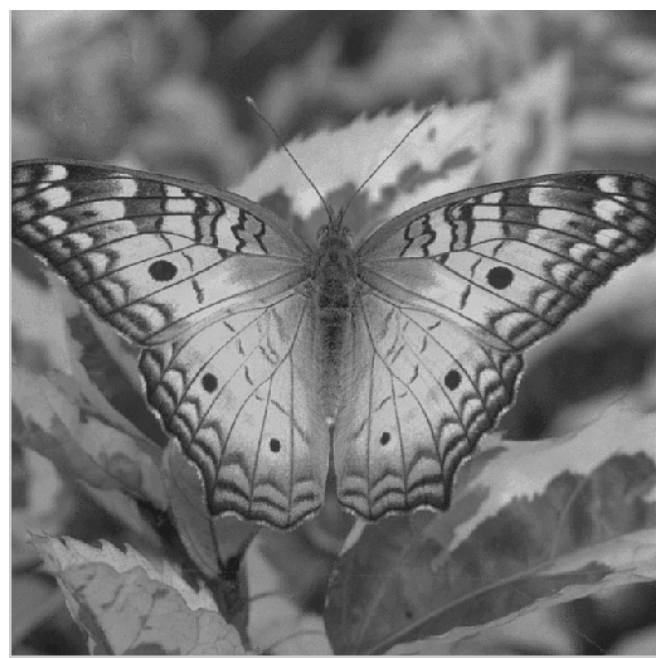

(j)

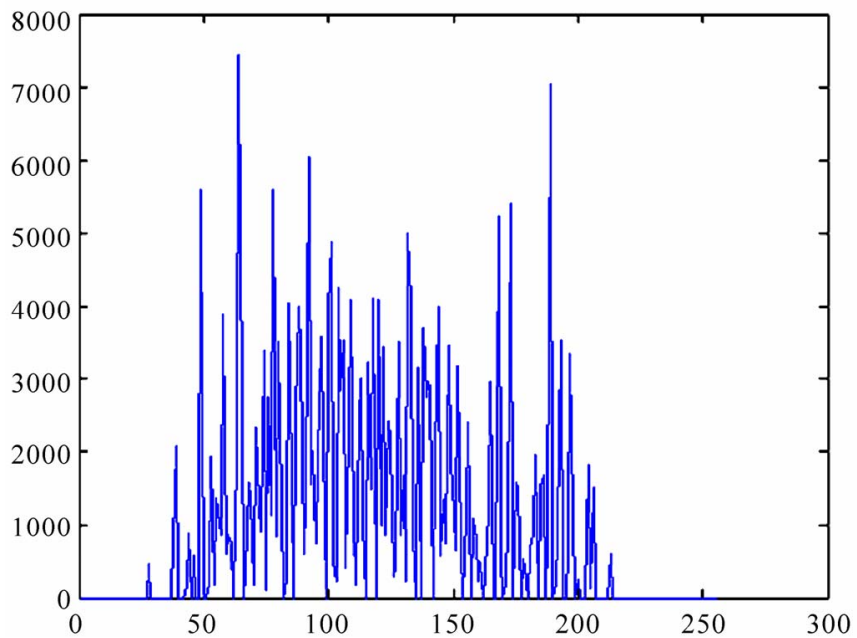

(j’)

Figure 1. Test Images and their histograms (a) Lena, (b) Pepper, (c) Baboon, (d) Hunter, (e) Map, (f) Cameraman, (g) Living room, (h) House,(i) Airplane, (j) Butterfly

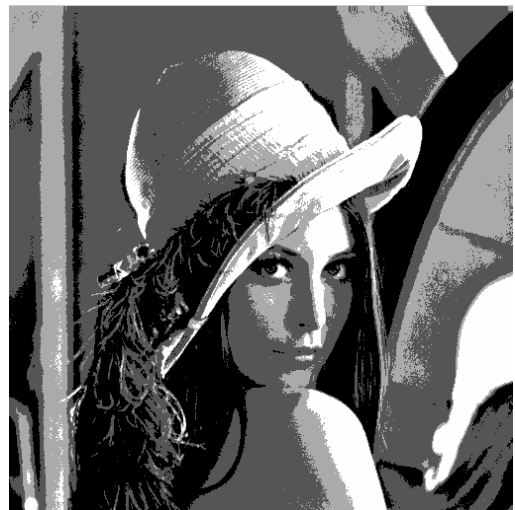

(a)

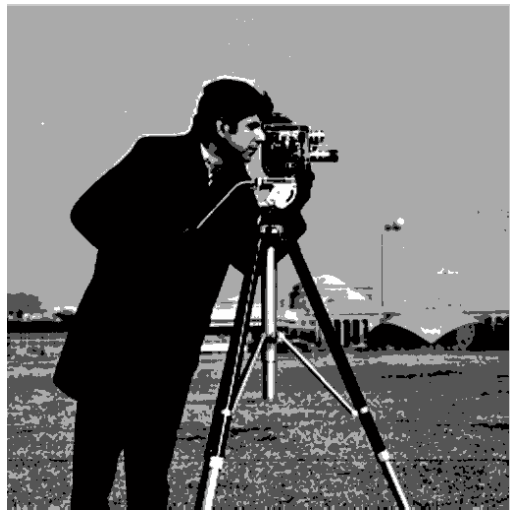

(b)

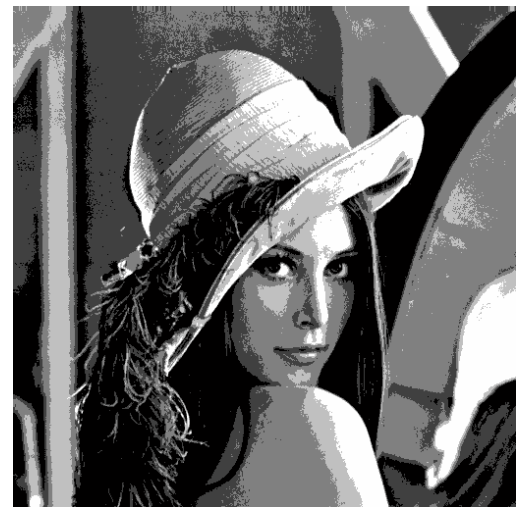

(a')

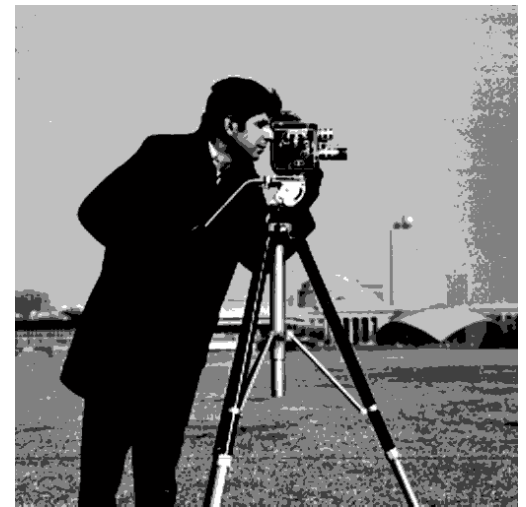

(b')

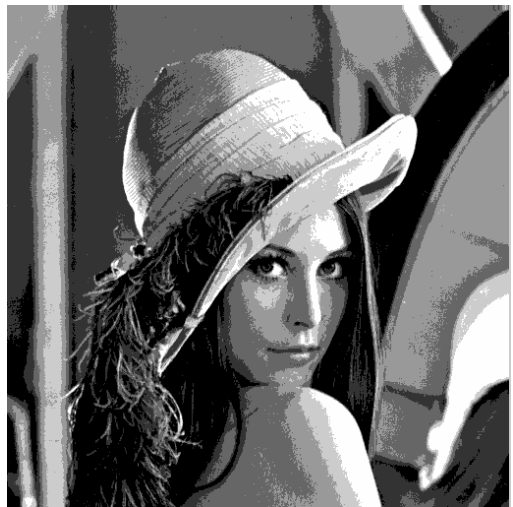

(a")

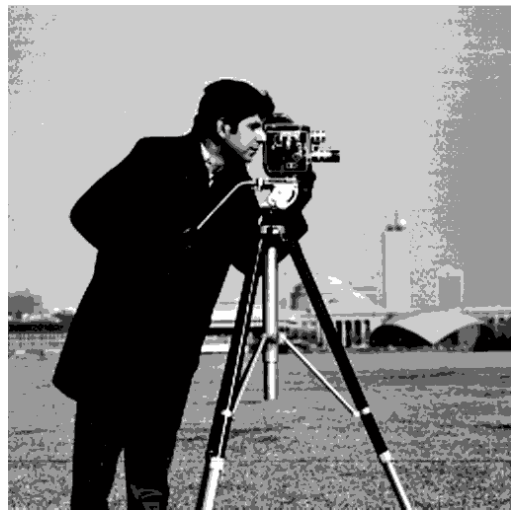

(b")

Figure 2. Thresholded images obtained by Kapur-PSO method ((a), (b) represents 3-level thresholding, (a'), (b’) represents 4-level thresholding, (a’), (b’’) represents 5-level thresholding)

and Otsu based methods has been evaluated in Tables 3 and 4 . The tables show the number of thresholds and the optimal threshold values with the corresponding objec- tive value for PSO and GA methods. It is observed from the table that in each case, the PSO could perform well as compared with the GA method. These two methods use 
Table 3. Comparison of optimal threshold values and objective values obtained by Kapur method

\begin{tabular}{|c|c|c|c|c|c|}
\hline \multirow{2}{*}{ Test Images } & \multirow{2}{*}{$\mathrm{m}$} & \multicolumn{2}{|c|}{ Optimal threshold values } & \multicolumn{2}{|c|}{ Objective values } \\
\hline & & PSO & GA & PSO & GA \\
\hline \multirow{4}{*}{ LENA } & 2 & 99,165 & 104,167 & 12.3459 & 12.3344 \\
\hline & 3 & $86,151,180$ & $72,151,180$ & 15.1336 & 14.9956 \\
\hline & 4 & $92,129,162,191$ & $57,110,178,184$ & 17.8388 & 17.0892 \\
\hline & 5 & $74,115,145,170,197$ & $96,112,151,186,198$ & 20.4427 & 19.5492 \\
\hline \multirow{4}{*}{ PEPPER } & 2 & 79,146 & 82,146 & 12.5168 & 12.5133 \\
\hline & 3 & $104,141,180$ & $108,127,186$ & 15.0939 & 14.7122 \\
\hline & 4 & $57,110,162,199$ & $72,102,172,204$ & 18.0974 & 17.6959 \\
\hline & 5 & $70,116,138,166,200$ & $77,107,124,178,209$ & 20.7338 & 20.0691 \\
\hline \multirow{4}{*}{ BABOON } & 2 & 76,144 & 93,152 & 12.2134 & 12.1847 \\
\hline & 3 & $72,130,181$ & $64,151,181$ & 15.0088 & 14.7457 \\
\hline & 4 & $65,121,153,180$ & $90,106,152,188$ & 17.5743 & 16.9356 \\
\hline & 5 & $73,110,142,166,192$ & $96,126,150,172,197$ & 20.2245 & 19.6622 \\
\hline \multirow{4}{*}{ HUNTER } & 2 & 83,179 & 75,178 & 12.3708 & 12.3496 \\
\hline & 3 & $85,128,166$ & $70,148,167$ & 15.1286 & 14.8381 \\
\hline & 4 & $74,131,174,200$ & $64,100,189,200$ & 18.0401 & 17.3189 \\
\hline & 5 & $90,120,164,190,219$ & $87,96,128,196,213$ & 20.5339 & 19.5635 \\
\hline \multirow{4}{*}{ MAP } & 2 & 97,181 & 84,174 & 4.9789 & 4.9610 \\
\hline & 3 & $74,140,181$ & $62,94,156$ & 5.5030 & 5.1351 \\
\hline & 4 & $92,128,152,207$ & $96,113,186,218$ & 5.6903 & 5.0740 \\
\hline & 5 & $66,109,121,150,195$ & $85,114,159,192,211$ & 5.9165 & 5.4302 \\
\hline \multirow{4}{*}{ CAMERAMAN } & 2 & 115,196 & 76,195 & 12.2595 & 11.9414 \\
\hline & 3 & $96,138,191$ & $111,165,189$ & 15.2110 & 14.8278 \\
\hline & 4 & $77,116,151,202$ & $71,80,141,192$ & 18.0009 & 17.1665 \\
\hline & 5 & $64,95,121,156,198$ & $66,110,169,180,209$ & 20.9631 & 19.7950 \\
\hline \multirow{4}{*}{ LIVINGROOM } & 2 & 86,175 & 84,171 & 12.4000 & 12.3923 \\
\hline & 3 & $73,158,187$ & $74,138,160$ & 15.2123 & 14.9700 \\
\hline & 4 & $59,124,172,202$ & $74,137,164,175$ & 18.1410 & 17.2063 \\
\hline & 5 & $72,97,119,158,197$ & $60,120,148,155,200$ & 20.6752 & 19.8410 \\
\hline \multirow{4}{*}{ HOUSE } & 2 & 81,144 & 91,145 & 10.8321 & 10.7436 \\
\hline & 3 & $81,116,155$ & $96,134,164$ & 13.1006 & 12.8473 \\
\hline & 4 & $75,123,154,193$ & $83,135,170,193$ & 15.1027 & 14.6588 \\
\hline & 5 & $48,97,139,159,189$ & $81,107,132,157,189$ & 17.2517 & 16.9452 \\
\hline \multirow{4}{*}{ AIRPLANE } & 2 & 80,175 & 90,176 & 12.1503 & 12.1153 \\
\hline & 3 & $72,121,191$ & $75,110,199$ & 15.2925 & 14.8059 \\
\hline & 4 & $74,129,162,188$ & $87,124,154,187$ & 18.0300 & 17.8923 \\
\hline & 5 & $81,118,144,167,192$ & $95,121,141,151,196$ & 20.3964 & 19.4465 \\
\hline \multirow{4}{*}{ BUTTERFLY } & 2 & 95,141 & 93,142 & 10.4743 & 10.4707 \\
\hline & 3 & $63,126,172$ & $96,103,167$ & 12.3130 & 11.6280 \\
\hline & 4 & $71,113,162,184$ & $111,149,155,173$ & 14.2317 & 13.3144 \\
\hline & 5 & $92,116,142,157,182$ & $75,105,140,179,198$ & 16.3374 & 15.7566 \\
\hline
\end{tabular}


Table 4. Comparison of optimal threshold values and objective values obtained by Otsu method

\begin{tabular}{|c|c|c|c|c|c|}
\hline \multirow{2}{*}{ Test Images } & \multirow{2}{*}{$\mathrm{m}$} & \multicolumn{2}{|c|}{ Optimal threshold values } & \multicolumn{2}{|c|}{ Objective values } \\
\hline & & PSO & GA & PSO & GA \\
\hline \multirow{4}{*}{ LENA } & 2 & 94,152 & 91,149 & 1961.4149 & 1960.9603 \\
\hline & 3 & $79,127,170$ & $80,124,173$ & 2127.7771 & 2126.4107 \\
\hline & 4 & $78,112,134,175$ & $80,126,159,185$ & 2180.6868 & 2173.7148 \\
\hline & 5 & $79,110,140,167,188$ & $80,116,146,179,213$ & 2212.5555 & 2196.2745 \\
\hline \multirow{4}{*}{ PEPPER } & 2 & 76,144 & 84,144 & 2469.5788 & 2457.1517 \\
\hline & 3 & $72,124,171$ & $65,116,175$ & 2623.2739 & 2614.0841 \\
\hline & 4 & $57,92,130,172$ & $62,108,142,177$ & 2695.8867 & 2682.8391 \\
\hline & 5 & $56,84,115,150,179$ & $52,90,128,166,191$ & 2733.5097 & 2725.8750 \\
\hline \multirow{4}{*}{ BABOON } & 2 & 96,149 & 98,151 & 1547.9977 & 1547.6588 \\
\hline & 3 & $85,126,166$ & $86,125,155$ & 1635.3623 & 1633.5220 \\
\hline & 4 & $79,105,140,174$ & $82,122,146,173$ & 1684.3363 & 1677.7052 \\
\hline & 5 & $74,104,134,161,180$ & $73,106,140,167,199$ & 1712.9582 & 1699.3909 \\
\hline \multirow{4}{*}{ HUNTER } & 2 & 52,116 & 51,115 & 3064.0688 & 3064.0156 \\
\hline & 3 & $39,86,135$ & $36,89,133$ & 3212.0585 & 3211.7947 \\
\hline & 4 & $36,84,130,157$ & $39,93,142,163$ & 3257.1767 & 3231.1313 \\
\hline & 5 & $37,85,125,154,177$ & $39,94,130,169,204$ & 3276.3173 & 3244.7387 \\
\hline \multirow{4}{*}{ MAP } & 2 & 113,177 & 81,173 & 2340.3950 & 2252.3864 \\
\hline & 3 & $81,145,197$ & $83,132,181$ & 2526.3034 & 2503.7932 \\
\hline & 4 & $92,133,162,206$ & $90,110,158,204$ & 2618.4894 & 2617.9534 \\
\hline & 5 & $79,116,139,162,204$ & $68,106,138,170,214$ & 2665.4116 & 2660.8599 \\
\hline \multirow{4}{*}{ CAMERAMAN } & 2 & 71,143 & 72,145 & 3609.3703 & 3609.0761 \\
\hline & 3 & $71,134,166$ & $71,143,196$ & 3677.1783 & 3643.2153 \\
\hline & 4 & $65,121,147,172$ & $59,119,155,203$ & 3722.6447 & 3710.7311 \\
\hline & 5 & $45,78,121,146,172$ & $51,106,141,167,194$ & 3764.9571 & 3755.5529 \\
\hline \multirow{4}{*}{ LIVINGROOM } & 2 & 88,145 & 89,155 & 1627.7966 & 1627.0537 \\
\hline & 3 & $81,127,165$ & $83,132,174$ & 1757.4664 & 1748.6885 \\
\hline & 4 & $69,110,143,178$ & $71,116,150,182$ & 1822.1136 & 1816.0692 \\
\hline & 5 & $56,98,128,156,190$ & $65,104,133,160,189$ & 1865.4766 & 1858.0959 \\
\hline \multirow{4}{*}{ HOUSE } & 2 & 57,127 & 56,124 & 3420.9868 & 3418.4387 \\
\hline & 3 & $48,104,165$ & $50,119,182$ & 3617.9836 & 3592.1268 \\
\hline & 4 & $40,88,140,194$ & $41,98,149,184$ & 3702.2895 & 3686.1240 \\
\hline & 5 & $32,74,129,158,188$ & $48,106,136,169,199$ & 3752.1468 & 3700.3010 \\
\hline \multirow{4}{*}{ AIRPLANE } & 2 & 117,174 & 116,175 & 1837.7222 & 1837.7144 \\
\hline & 3 & $99,158,193$ & $86,133,204$ & 1905.7664 & 1844.5642 \\
\hline & 4 & $84,125,168,201$ & $71,119,164,200$ & 1953.8872 & 1950.5919 \\
\hline & 5 & $60,101,138,177,204$ & $84,124,164,188,204$ & 1977.9742 & 1973.0894 \\
\hline \multirow{4}{*}{ BUTTERFLY } & 2 & 99,150 & 100,151 & 1553.0687 & 1552.4129 \\
\hline & 3 & $79,119,164$ & $74,115,155$ & 1665.7589 & 1662.6963 \\
\hline & 4 & $80,113,145,177$ & $82,119,154,184$ & 1702.9069 & 1696.6940 \\
\hline & 5 & $75,106,129,157,180$ & $77,107,134,171,185$ & 1730.7879 & 1716.0428 \\
\hline
\end{tabular}


Table 5. Comparison of standard deviation and CPU time (in seconds) for Kapur and Otsu methods

\begin{tabular}{|c|c|c|c|c|c|c|c|c|c|}
\hline \multirow{3}{*}{ Test Images } & \multirow{3}{*}{$\mathrm{m}$} & \multicolumn{4}{|c|}{ Standard Deviation } & \multicolumn{4}{|c|}{ Computation time } \\
\hline & & \multicolumn{2}{|c|}{ Kapur method } & \multicolumn{2}{|c|}{ Otsu method } & \multicolumn{2}{|c|}{ Kapur method } & \multicolumn{2}{|c|}{ Otsu method } \\
\hline & & PSO & GA & PSO & GA & PSO & GA & PSO & GA \\
\hline \multirow{4}{*}{ LENA } & 2 & 0.0033 & 0.0049 & 0.1423 & 0.2077 & 7.8594 & 8.5469 & 3.5781 & 3.9688 \\
\hline & 3 & 0.0390 & 0.1100 & 0.4155 & 0.5555 & 8.3594 & 8.8594 & 4.4031 & 5.2969 \\
\hline & 4 & 0.1810 & 0.2594 & 2.3601 & 3.0640 & 9.1719 & 9.5156 & 4.7500 & 5.6094 \\
\hline & 5 & 0.2181 & 0.3043 & 4.5341 & 5.7362 & 9.4063 & 10.1250 & 5.2031 & 5.8938 \\
\hline \multirow{4}{*}{ PEPPER } & 2 & 0.0012 & 0.0031 & 0.0956 & 0.1455 & 7.1358 & 8.6492 & 3.4010 & 3.8569 \\
\hline & 3 & 0.0764 & 0.1750 & 0.1629 & 0.2891 & 7.6250 & 9.1056 & 4.3125 & 4.9787 \\
\hline & 4 & 0.1080 & 0.2707 & 2.1102 & 3.9721 & 8.1254 & 9.6406 & 4.6719 & 5.5156 \\
\hline & 5 & 0.1758 & 0.3048 & 3.2057 & 4.9999 & 8.4844 & 9.9688 & 4.8125 & 5.9844 \\
\hline \multirow{4}{*}{ BABOON } & 2 & 0.0077 & 0.0567 & 0.1040 & 0.2224 & 8.0016 & 8.3563 & 3.8469 & 4.3969 \\
\hline & 3 & 0.0816 & 0.1580 & 0.5720 & 1.5317 & 8.7188 & 9.3750 & 4.3125 & 4.7969 \\
\hline & 4 & 0.0853 & 0.1765 & 2.1501 & 3.0653 & 9.1084 & 9.6750 & 4.9063 & 5.6094 \\
\hline & 5 & 0.1899 & 0.2775 & 3.4447 & 4.6721 & 9.7813 & 10.1875 & 5.3281 & 6.0109 \\
\hline \multirow{4}{*}{ HUNTER } & 2 & 0.0068 & 0.0148 & 0.2282 & 0.3283 & 8.000 & 8.6406 & 3.8438 & 4.4063 \\
\hline & 3 & 0.0936 & 0.1741 & 0.8203 & 1.8080 & 8.7031 & 9.9844 & 4.4844 & 4.8625 \\
\hline & 4 & 0.1560 & 0.2192 & 2.9836 & 6.3644 & 9.0313 & 9.6219 & 4.8125 & 5.3906 \\
\hline & 5 & 0.2720 & 0.3466 & 7.3030 & 11.1247 & 10.1406 & 10.6094 & 5.3031 & 6.1563 \\
\hline \multirow{4}{*}{ MAP } & 2 & 0.0023 & 0.0030 & 1.2241 & 1.8856 & 6.8906 & 7.4625 & 3.6094 & 4.2000 \\
\hline & 3 & 0.1153 & 0.1226 & 1.2298 & 2.1368 & 7.1563 & 7.6563 & 4.4219 & 4.9688 \\
\hline & 4 & 0.1366 & 0.1849 & 2.2333 & 4.5790 & 8.1250 & 8.9094 & 4.8750 & 5.5156 \\
\hline & 5 & 0.1521 & 0.1901 & 3.4511 & 6.3580 & 8.3594 & 9.7969 & 5.7500 & 6.4188 \\
\hline \multirow{4}{*}{ CAMERAMAN } & 2 & 0.1001 & 0.1270 & 0.0908 & 0.3812 & 8.4844 & 9.2500 & 3.4844 & 3.9531 \\
\hline & 3 & 0.1107 & 0.2136 & 6.3502 & 9.4711 & 9.0625 & 9.7000 & 4.1250 & 4.8125 \\
\hline & 4 & 0.2005 & 0.2857 & 2.4498 & 4.5059 & 9.1250 & 9.9844 & 4.7406 & 5.2500 \\
\hline & 5 & 0.2734 & 0.3528 & 8.9650 & 11.0079 & 10.1094 & 10.9688 & 5.2656 & 6.0025 \\
\hline \multirow{4}{*}{ LIVINGROOM } & 2 & 0.0022 & 0.0039 & 0.2637 & 0.5425 & 7.5844 & 8.2156 & 3.3281 & 3.7656 \\
\hline & 3 & 0.0718 & 0.1364 & 1.0446 & 2.4428 & 8.7188 & 9.6250 & 4.0469 & 4.9531 \\
\hline & 4 & 0.2286 & 0.3220 & 2.0787 & 3.0313 & 9.1001 & 9.7656 & 4.5000 & 5.1056 \\
\hline & 5 & 0.2619 & 0.3805 & 2.2655 & 4.3189 & 10.1719 & 10.5631 & 5.7969 & 6.6094 \\
\hline \multirow{4}{*}{ HOUSE } & 2 & 0.0224 & 0.0637 & 0.8001 & 1.7181 & 7.9063 & 8.3656 & 3.6252 & 4.4313 \\
\hline & 3 & 0.0805 & 0.1549 & 3.1018 & 6.2939 & 8.2626 & 9.2500 & 4.2969 & 4.9844 \\
\hline & 4 & 0.1324 & 0.2555 & 3.7038 & 8.2156 & 8.8438 & 9.5938 & 4.6094 & 5.3750 \\
\hline & 5 & 0.1824 & 0.2696 & 6.5478 & 9.9390 & 9.6406 & 10.0938 & 5.7344 & 6.6963 \\
\hline \multirow{4}{*}{ AIRPLANE } & 2 & 0.0106 & 0.0305 & 1.1731 & 2.7001 & 7.9844 & 8.7188 & 3.4688 & 4.0000 \\
\hline & 3 & 0.1248 & 0.1958 & 2.5107 & 5.0948 & 8.9688 & 10.4844 & 4.5938 & 5.1875 \\
\hline & 4 & 0.1424 & 0.3011 & 3.4728 & 7.0157 & 9.2031 & 9.9531 & 4.7969 & 5.3594 \\
\hline & 5 & 0.2760 & 0.3369 & 4.7571 & 8.6500 & 9.9688 & 10.4031 & 5.0781 & 5.8125 \\
\hline \multirow{4}{*}{ BUTTERFLY } & 2 & 0.0025 & 0.0872 & 1.6744 & 2.3493 & 7.7188 & 8.4906 & 3.5313 & 3.9219 \\
\hline & 3 & 0.1880 & 0.2021 & 2.2356 & 3.4016 & 8.5469 & 9.4656 & 4.1875 & 4.9531 \\
\hline & 4 & 0.2473 & 0.2596 & 4.2227 & 5.2383 & 9.0000 & 9.8659 & 4.8281 & 5.5156 \\
\hline & 5 & 0.2821 & 0.3977 & 5.1212 & 6.2719 & 9.3813 & 10.2469 & 5.4594 & 6.1313 \\
\hline
\end{tabular}




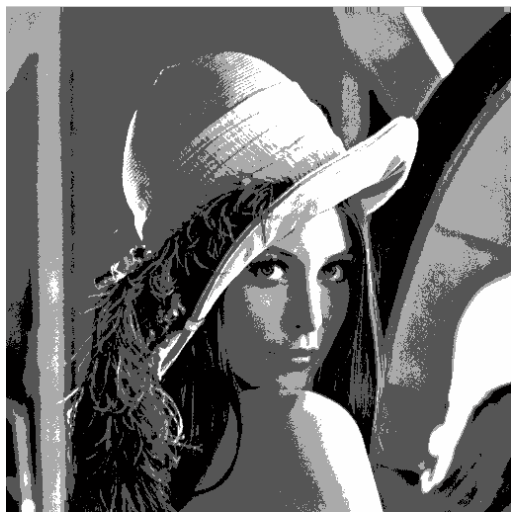

(a)

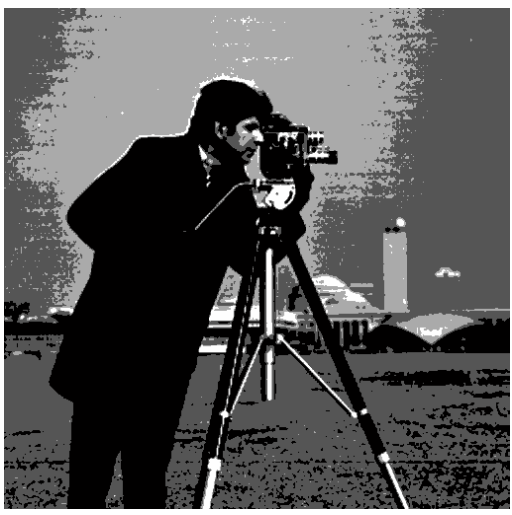

(b)

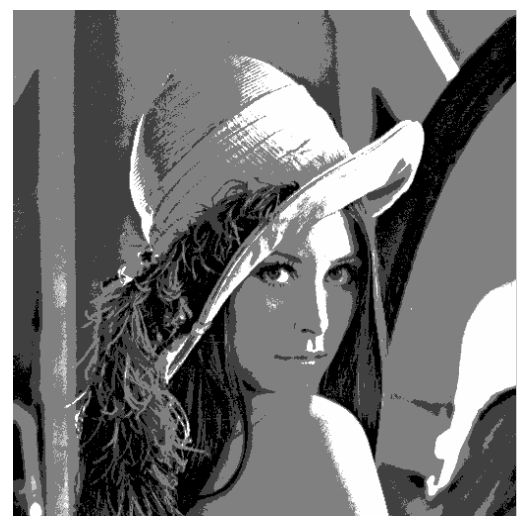

(a')

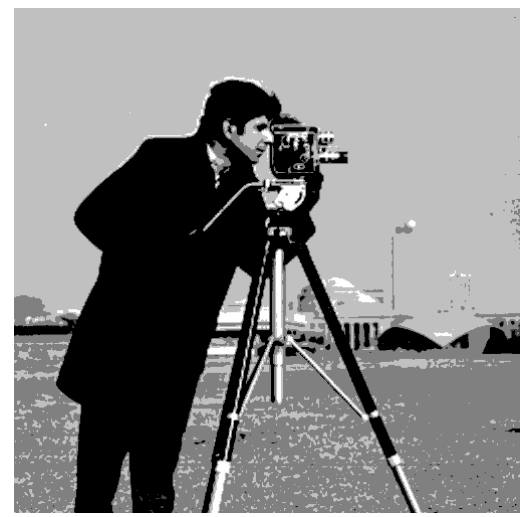

(b')

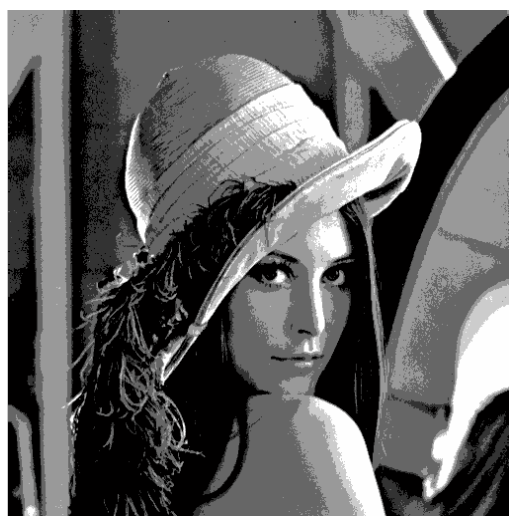

(a")

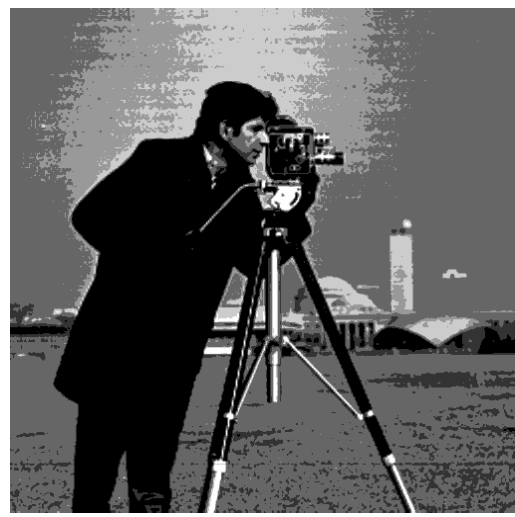

(b")

Figure 3. Thresholded images obtained by Otsu-PSO method ((a), (b) represents 3-level thresholding, (a'), (b’) represents 4-level thresholding, (a”), (b’') represents 5-level thresholding)

the objective function to decide whether the number of thresholds has reached the optimal value or not. The higher value of the objective function results in better segmentation.

For a visual interpretation of the segmentation results, the segmented lena and cameraman images for both Kapur-PSO and Otsu-PSO with $\mathrm{m}=3,4$ and 5 are presented in Figures 2 and $\mathbf{3}$ respectively. It can be easily seen that the quality of segmentation is better, in each case, when $m=5$ is chosen.

The standard deviation values and computation time obtained from Kapur and Otsu based evolutionary algorithms are given in Table 5. The higher value of standard deviation shows that the results of experiment are unstable. From the tables, it is seen that the PSO method is more stable than the GA method. It is also observed from the table that, even though the Kapur-based method gives lower standard deviation than the Otsu's method, the computation time of Kapur based PSO is higher than the Otsu based PSO.

\section{Conclusions}

In this paper, particle swarm optimization (PSO) based multilevel thresholding has been presented for image segmentation. In order to verify the efficiency and effectiveness of the proposed PSO approach, ten standard test images are investigated. The performance of this approach has been compared with the GA method, and it is found that PSO outperforms GA approach in terms of solution quality, convergence and robustness. Compared with all the cases, the Kapur-PSO gives lower standard deviation value. Even though the Kapur-PSO gives lower standard deviation, the Otsu-PSO method converges quickly than the Kapur method. Hence, the Otsu-PSO approach is an efficient tool for finding optimized threshold values.

\section{REFERENCES}

[1] J. K. Udupa and S. Samarasekera, "Fuzzy Connectedness and Object Definition: Theory, Algorithms and Applications in Image Segmentation," Graphical Models and Image Processing, Vol. 58, No. 3, 1996, pp. 246-261.

[2] P. K. Sahoo, S. Soltani and A. K. C. Wong, "A Survey of Thresholding Techniques," Computer Vision, Graphics and Image Processing, Vol. 41, No. 2, 1998, pp. 233-260.

[3] N. P. Pal and S. K. Pal, "A Review on Image Segmenta- 
tion Techniques," Pattern Recognition, Vol. 26, No. 9, 1993, pp. 1277-1294.

[4] M. Sezgin and B. Sankar, "Survey over Image Thresholding Techniques and Quantitative Performance Evaluation," Journal of Electronic Imaging, Vol. 13, No. 1, 2004, pp. 146-165.

[5] S. U. Lee, S. Y. Chung and R. H. Park, "A Comparative Performance Study of Several Global Thresholding Techniques for Segmentation," Computer Vision, Graphics and Image Processing, Vol. 52, No. 2, 1990, pp. 171-190.

[6] N. Otsu, "A Threshold Selection Method from GrayLevel Histograms," IEEE Transaction on Systems, Man and Cybernetics, Vol. 9, No. 1, 1979, pp. 62-66.

[7] W. Tao, H. Jin and L. Liu, "Object Segmentation Using Ant Colony Optimization Algorithm and Fuzzy Entropy," Pattern Recognition Letters, Vol. 28, No. 7, 2007, pp. 788-796.

[8] O. J. Tobias and R. Seara, "Image Segmentation by Histogram Thresholding Using Fuzzy Sets," IEEE Transaction on Image Processing, Vol. 11, No. 12, 2002, pp. 1457-1465.

[9] J. N. Kapur, P. K. Sahoo and A. K. C. Wong, "A New Method for Gray-Level Picture Thresholding Using the Entropy of The Histogram," Computer Vision, Graphics and Image Processing, Vol. 29, No. 3, 1985, pp. 273-285.

[10] T. Pun, "Entropy Thresholding: A New Approach," Computer Vision, Graphics and Image Processing, Vol. 16, No. 3, 1981, pp. 210-239.

[11] A. D. Brink, "Minimum Spatial Entropy Threshold Selection," IEEE Proceedings, Vision Image and Signal Processing, Vol. 142, No. 3, 1995, pp. 128-132.

[12] X. Li, Z. Zhao and H. D. Cheng, "Fuzzy Entropy Threshold Approach to Breast Cancer Detection," Information Sciences, Vol. 4, No. 1, 1995, pp.49-56.

[13] L. K. Huang and M. J. Wang, "Image Thresholding by Minimizing the Measure of Fuzziness," Pattern Recognition, Vol. 28, No. 1, 1995, pp. 41-51.

[14] J. Kittler and J. Illingworth, "Minimum Error Thresholding," Pattern Recognition, Vol. 19, No. 1, 1986, pp. 41-47.

[15] Q. Ye and P. Danielsson, "On Minimum Error Thresholding and its Implementation," Pattern Recognition Letters, Vol. 7, No. 4, 1988, pp. 201-206.

[16] U. Gonzales-Baron and F. Butler, "A Comparison of Seven Thresholding Techniques with the K-Means Clustering Algorithm for Measurement of Bread-Crumb Features by Digital Image Analysis," Journal of Food Engi- neering, Vol. 74, No. 2, 2006, pp. 268-278.

[17] P. Y. Yin and L. H. Chen, "A Fast Iterative Scheme For Multilevel Thresholding Methods," Signal Processing, Vol. 60, No. 3, 1997, pp. 305-313.

[18] P. S. T. Liao, S. Chen and P. C. Chung, "A Fast Algorithm for Multilevel Thresholding," Journal of Information Science and Engineering, Vol. 17, No. 5, 2001, pp. 713-727.

[19] K. C. Lin, "Fast Image Thresholding by Finding Zero(S) of the First Derivative of between Class Variance," $M a-$ chine Vision and Applications, Vol. 13, No. 5-6, 2003, pp. 254-262.

[20] P.-Y. Yin and L.-H. Chen, "A Fast Iterative Scheme for Multilevel Thresholding Methods," Signal Processing, Vol. 60, No. 3, 1997, pp. 305-313.

[21] E. Cuevas, D. Zaldivar and M. Perez-Cisneros, "A Novel Multi-Threshold Segmentation Approach Based on Differential Evolution Optimization," Expert Systems with Applications, Vol. 37, No. 7, 2010, pp. 5265-5271.

[22] W. B. Tao, H. Jin and L. M. Liu, "Object Segmentation Using Ant Colony Optimization Algorithm and Fuzzy Entropy," Pattern Recognition Letters, Vol. 28, No. 7, 2008, pp. 788-796.

[23] P. Y. Yin, "A Fast Scheme for Optimal Thresholding Using Genetic Algorithms,” Signal Processing, Vol. 72, No. 2, 1999, pp. 85-95.

[24] C. C. Lai and D. C. Tseng, "A Hybrid Approach Using Gaussian Smoothing and Genetic Algorithm for Multilevel Thresholding," International Journal of Hybrid Intelligent Systems, Vol. 1, No. 3, 2004, pp. 143-152.

[25] D. B. Fogel, "Evolutionary Computation: Toward a New Philosophy of Machine Intelligence," 2nd Edition, IEEE Press, Piscataway, 2000.

[26] J. Kennedy and R. Eberhart, "Particle Swarm Optimization," Proceedings of the IEEE Conference on Neural Networks-ICNN'95, Perth, Vol. 4, 1995, pp. 1942-1948.

[27] Y.-T. Kao, E. Zahara and I-W. Kao, "A Hybridized Approach to Data Clustering," Expert Systems with Applications, Vol. 34, No. 3, 2008, pp. 1754-1762.

[28] Z.-J. Lee, S.-W. Lin, S.-F. Su and C.-Y. Lin, "A Hybrid Watermarking Technique Applied to Digital Images," Expert Systems with Applications, Vol. 8, No. 1, 2008, pp. 789-808

[29] C.-C. Tseng, J.-G. Hsieh and J.-H. Jeng, "Fractal Image Compression Using Visual-Based Particle Swarm Optimization," Image and Vision Computing, Vol. 26, No. 8, 2008, pp. 1154-1162. 